\title{
A proposal for using UAS in radio navigation aids flight inspection
}

\author{
Jorge Ramírez ${ }^{1}$, Cristina Barrado ${ }^{2,}$ Enric Pastor $^{3}$ and J.C Garcia ${ }^{4}$ \\ Technical University of Catalonia, Castelldelfels, Barcelona 08860, Spain
}

\begin{abstract}
The main objective of this paper is to contribute to the inherent necessity of the aerial transport system to guarantee the safety of its users in an efficient manner. This efficiency is required to accomplish the objective of transport people and goods with cost content enough to allow the access to this system at the biggest number of users. To improve this efficiency we propose a new paradigm in Flight Inspection Systems. This new paradigm separates the flight inspection platform in two separate segments, remaining in the air segment the strictly needed resources, the sensors, by mean of Unmanned Aerial Systems (UAS).
\end{abstract}

\section{Introduction}

There are a multitude of factors converging in the aerial transport system which contribute to the achievement of safety levels, adequate to commercial exploitation. Those safety levels are in concordance with the standards that the civil aviation community has adopted. Different levels of abstractions (materials, systems, procedures, operations, organizations...) are given in which safety maintenance and safety promotion are essential.

Identification of present position and current course shall be done in an homogeneous manner and in accordance with the exigency of each flight phase. The means currently used for navigation are the Radio Navigation Aids (NavAids). Those NavAids could be seen as radio-frequency emitters. Their emission structure and geographic location allows the users (the flying aircrafts) to compute their position and course in a precise way.

Current society requires to the aerial transport system (since decades ago) the capability to fly in a safe manner, even in non favorable visibility conditions like night flights, in presence of fog or among the clouds. This requirement makes the use of Radio Navigation Aids critical for the Aerial Transport System.

Those NavAids, as electrical, electronic, radio-frecuency artifacts that they are, could present a biased behavior. The contention of this bias among acceptable limits for its use becomes critical for the Navigation Services users as the physical integrity, of the aircraft frame and its crew and passengers, depends on the appropriate behavior of the Radio Navigation Aids.

To ensure the proper functioning of these NavAids different mechanisms are used. The first and most obvious is to ensure the design, manufacture and installation in accordance with standards of quality assurance that are the norm in the aviation community (SAE ARP 4754 [SAE_1], SAE ARP 4761 [SAE_2], RTCA 178B [RTCA_1], RTCA 278 [RTCA_2], ARINC standards ...)

A complementary way of ensuring the adequate performances of the NavAids is the Flight inspection. This time the focus is on the monitoring of the performances, not for the adequacy of the design.

The Flight Inspection shall demonstrate that the performances of the NavAid is are confined to a standard behavior and that their accuracy is the required for approval by the competent authorities. Such approval could be assimilated with obtaining the permission to fly of an aircraft while the adequacy of the design and manufacture of

\footnotetext{
1 Assistant Lecturer, Castelldefels School of technology, room 119 building C3 Av. Del Canal Olímpic 15 cp08860 / jorge.ramirez@upc.edu

2 Professor, Castelldefels School of technology, room 013P building C4 Av. Del Canal Olímpic 15 cp08860 / cristina.barrado@ac.upc.edu

3 Professor, Castelldefels School of technology, room 013P building C4 Av. Del Canal Olímpic 15 cp08860 / cristina.barrado@ac.upc.edu

${ }^{4}$ Student, Castelldefels School of technology

American Institute of Aeronautics and Astronautics 092407
} 
the NavAid could be assimilated with the certification of aircraft type and design organization or production organization certification.

Since the functional objective of a NavAid is to provide a radio-frequency emission with a known structure (spectrum, timing, power...) in order to identify this emission with the known site position, to demonstrate that its behavior is confined to a standard behavior it shall be demonstrated that this emission corresponds with the standard. To achieve this demonstration, a set of parameters shall be measured from the point of view of the final user. I other words, they should be measured from the air (which is the place where they are used).

Such monitoring is carried out in each of the following circumstances:

1. Before starting the operations of the NavAid to ensure that it works properly before allowing users to use.

2. After conducting an scheduled maintenance work, to ensure that the operations has not had any adverse impact on the operation of the NavAid.

3. After some unscheduled interruption of service, to ensure that this return to the operation was successful.

4. From a regular basis to ensure that there are no deviations from the standard operation.

A recommendation of the magnitudes to be observed and its associated periods of supervision can be found in the ICAO documents [ICAO_1]. That document sets out a series of recommendations which, although not reach the level of standards or recommended practices, reflects actual practices of the ICAO member states during NavAids tests (both in ground and in flight).

The use of aircrafts for flight inspection of NavAids provides the authorities with the magnitude to be inspected measured in the same place that they are used but it has a big inconvenience: its price.

This proposal has as its main contribution the optimization of flight testing using UAS technology to separate the, usually indivisible, platform in two segments:

- Air Segment.

- Land Segment.

\section{State of the art}

In assessing the state of the art in the in-flight inspection shall be considered the fragmentation of the market. The Single European Sky (SES) initiative has as main objective to integrate the airspace of the European countries. While some aspects have managed to unite some time ago (e.g: certifications for aircraft type, design organization certificates, production organization certificates), others persist as dependent of each European country.

The certification of NavAids (and the in-flight inspection as part of) is one of these aspects that have not been properly consolidated. There is a process for normalization of ATM conducted by the European Aviation Safety Agency (EASA) which published a Notice of Proposed Amendment (NPA) [EASA_1] stating its intention of assuming the regulation competencies for ATM.

This fragmented scenario favors the use of generic flying means in order to content the development costs into limit affordable to the Navigation Service Providers: a type of aircraft available on the market and the required instrumentation.

These platforms shall be used to inspect the different types of NavAids over their respective geographic locations. A logical conclusion is to use the aircraft itself to transport the crew, the flight inspector and the different instrument needed for ground inspection.

The previous conclusion is even more clear when considering that flight inspection starts so far as 1920's [9] when current Information Processing and Communication where just science fiction, circumstance that justifies the processing of the flight inspection on board.

ICAO proposes in its document [ICAO_1], some recommendations for the flight inspection including magnitudes to be observed, procedures, aircrafts and systems. This recommendations are an abstraction set of recommendations derived from the contributions of the different ICAO states members that conducts this flight inspection activities. Each of this nations have its own regulation for flight inspection e.g: USA has its standar flight inspection Manual [FAA_1]. In the case of Europe, EASA has the intention of assuming the competencies but currently each European Nation has its own Flight Inspection Regulation.

This paper will focus on the aircraft needed for flight inspection, the impact of these recommendations on it and how the UAS technology could improve Flight Inspection systems.

Among the aircraft recommendations, it is specially constraining the suggestion of selecting aircrafts big enough to transport equipments and personnel. This recommendation (which is clearly different than a requirement) leads us to the current contradiction of using large-capacity aircrafts, when a flight inspection consist in to carry a few 
sensors to spatial location in order to measure some electromagnetic magnitudes. This architecture is summarized in following fig. 1:

- Air Segment. Anything needed for the flight inspection shall be carried in the aircraft. This includes of course the sensors, the position determination system, the data recording system (or data logging) but also the flight inspector himself, the pilots, their seats, enough room for them air conditioning system, toilets, room for extra equipment...etc. Basically the antennae and the receivers of the different Navaids that are inspected (e.g: VOR, DME, ILS). Its function is to measure the values of the Navaid in the air.

O Position Determination System. To verify the adequacy of the measured values to the standard, each value needs to be related to the position in which was measured. Usually it is composed by a Satellite based navigation system but can also include other means as teodolites.

Flight Control. By Flight Control it is intended the operations that a pilot has to do in a manned aircraft to operate in the airspace.

Mission Control. The control of the Flight Inspection to ensure that the required data are collected and that the flight inspection has been conducted properly.

Data Logging. To comply with the ICAO recommendations it is mandatory the storage of the telemetry and instruments during the flight.

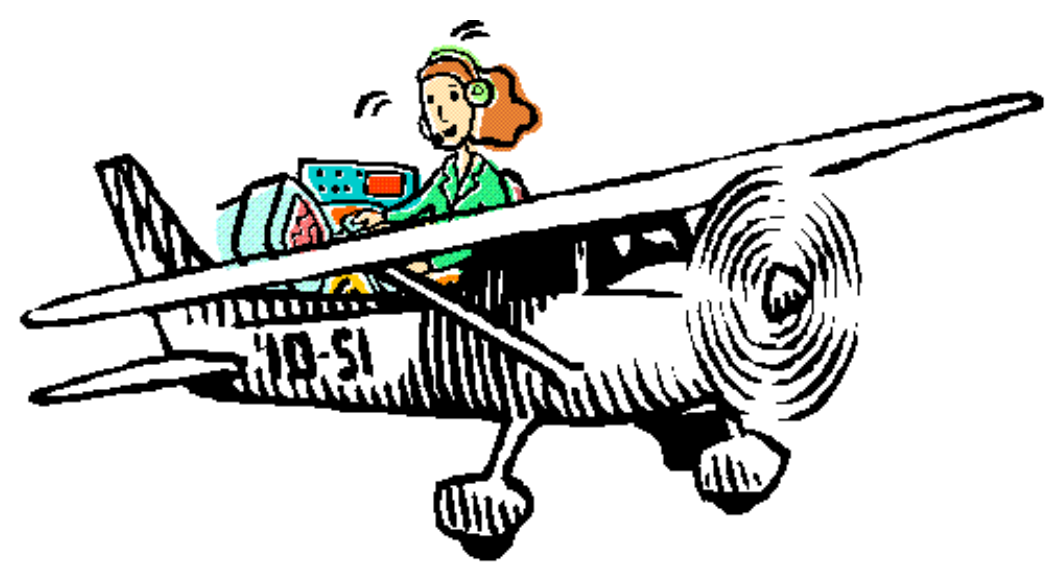

\begin{tabular}{|c|c|}
\hline \multicolumn{2}{|c|}{$\begin{array}{l}\text { Inspection } \\
\text { Platform }\end{array}$} \\
\hline $\begin{array}{l}\text { Flight } \\
\text { Control }\end{array}$ & $\begin{array}{l}\text { Mission } \\
\text { Control }\end{array}$ \\
\hline Sensors & $\begin{array}{l}\text { Position } \\
\text { Det. }\end{array}$ \\
\hline $\begin{array}{l}\text { Data } \\
\text { Logging }\end{array}$ & \\
\hline
\end{tabular}

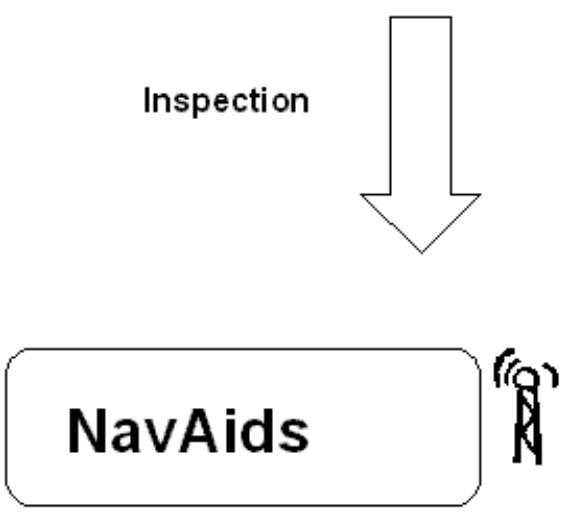

Figure 1: Current Flight Inspection System architecture

In the Flight Inspection community there is a trend to systems providing more flexibility. In [Qvist_1] we can found a proposal to locate the flight inspection down the aircraft and to maintain a telecom contact with the pilots in order to allow modifications to the initial flight plan.

In [Wede_1] there are different considerations about the possibility of keeping the flight inspector on ground, using more integrated an miniaturized Flight Inspection Systems and data links. 
The development of Air Transport System (ATS) new technologies is essential to ensure the development and enhancement of its capabilities, currently congested and near collapse in some areas of the world. In Europe SESAR consortium has planned a work program for conducting the research needed to implement the required improvements [SESAR_1]. This work program is currently in its definition phase which precedes the implementation phase that will extent until the 2020s.

From the operational perspective of any flight we can observe that the responsibility of eventual incidents is shared among:

Air Traffic Control which is responsibility of the Air Traffic Controllers (ATC). This operation is performed over voice communications in which the codification is made in Natural Language.

Aircraft Safety which is responsibility of the Pilot in Command of the Aircraft.

This current dichotomy represents major key factors limiting the integration of UAS into the airspace, the interface between ATC and UAS and the safety management during UAS operations.

The concepts and methodologies raised in the projects UAVnet [UAVNET_1] and IFATS [IFATS_1], as well as previous projects like UAV Safety Issues for Civil Operations (USICO) may be critical to define a strategy of integration of UAS in civil airspace.

UAVnet [UAVNET_1] is a thematic network born of a European project dedicated to promoting progress in the development of UAS for civilian use. Its objective is to promote and develop technologies for UAS in non-military tasks, where a key factor is to improve the safety of flights of unmanned aircraft within the civil sphere. Fruit of this project has been generated:

- Recommendations for the certifications of Air Navigation Systems.

- Recommendations for regulating the operations of UAS

- See and Avoid technologies (Activa Surveillance).

- Image recognition, sensors and technologies adapted to ADS-B.

- UAS Flight simulations.

IFATS [IFATS_1] is a project devoted to innovation in the future air transport system and proposes to study a revolutionary concept for air control system of the future: To automate the maximum navigation capabilities of the aircraft, providing it with autonomy and decision capability. The key requirement of the project is to improve the ATS efficiency while ensuring at all times its safety.

According to the proposals of the UAVNET group in its road map "European Civil Unmanned Air Vehicle Roadmap" [UAVNET_1], Europe must establish strategic lines for the long-term and constitute a center of excellence that includes the coordination of actors devoted to research on civilian UAS. The strategy proposed by European Comission has been named STAR21[EAC_1] and contemplates the deployment of UAS during the period from 2010 to 2015.

Thus we now find:

In one hand, an inspection technology heir to that used for decades [ICAO_1]. It is safe, reliable, sturdy but using some means excessively artisans and over dimensioned.

In the other hand we have an emergent technology as UAS is which could provide a more efficient mean for flight inspection

In the middle of both technologies a need from the In-flight inspection of more flexibility for the the operations and the systems.

The Volpe Center shown some vulnerabilities of the GPS [Volpe_1] that discarded the use of emerging Satellite Navigation technologies (notably GPS or Galileo) as sole Mean of Navigation. Nevertheless the improvement in navigation thanks to these technologies make its commissioning a matter of time.

The decommissioning of old infrastructure is quite delicate as a backup for emerging technologies are required. This implies that, with the exception of NDB, there are no plans to decommissioning existing NavAids (See [EuroC_1]). The simultaneous commissioning of new technology NavAids and the non Decommissioning of Old Navaid [EuroC_1] together with the requirement of improve the efficiency in SESAR [SESAR_1]. projects the need for flight inspection systems from the present to the future and justifies the search for efficient flight inspection systems capable of conducting the calibration of both legacy and new technology Navaids.

The key stone of this project is the belief that some constraints, currently excessively restrictive, could be easily and efficiently implemented with the use of UAS technology. 


\section{OBJECTIVES}

This proposal has as its masterpiece the study of the technological and regulatory constraints of radionavigation flight inspection using the UAS technology. This use allows the separation of the flight inspection in two segments:

I.Air Segment. Includes the elements essentials in the air, particularly antennas, elements of measurement, collection /storage of data.

II.Ground Segment. Includes all those not strictly necessary for the ongoing inspection, electronic spare parts, as well as the capability to carry comfortably personnel and ground equipment.

Such separation could now be translated into proper use of telecommunications capabilities available to us so that the platform could be seen from the viewpoint of the mission, as a network of computers interacting in a common mission.

Thanks to the separation into two segments, the Air Segment could be resized, replacing the existing aircrafts (large, expensive of acquire and to maintain) by UAS adjusted to the real needs in Flight Inspection: to carry sensors in the area to observe. This would bring flights the inherent characteristics of these devices: lower cost of the platform, lower operating costs, increased availability, etc.. Benefits that would revert in an air transport system more affordable (lower costs per flight test).

An additional optimization resulting from the separation into two segments of the platform inspection, is the simultaneous use of several aerial vehicles reporting to the same Ground Segment station. By this provision different areas could be simultaneously inspected, reducing the number of coordinated actions with air navigation and decreasing the time that an area is disabled by being inspected. E.g. different runway headers in a single airport inspection.

The different technical objectives identified in this proposal are:

- To design an UAS payload able to inspect Radio Navigation Aids both day and night.

- To design a mechanism for transmitting this information to a base station (from Air segment to Ground Segment) in real time or near real time without lost of information in case of communications link lost.

- To develop systems that use precise positioning systems complementary to those that are inspected.

- To design a mission planning system that allows its efficient use and exploitation by non-specialist.

- Integration of all the systems in a Flight inspection system.

\section{Requirement Review}

To demonstrate the adequacy of UAS technology to flight inspection, the desirable characteristics of an aircraft devoted to Flight Inspection (as listed in [ICAO_1], adjoint 1 to chapter 1, "Flight Inspection Aircraft") are commented:

a) The adequate reliability, is given by the use of cells, autopilot system and engine already developed by other companies. The efficiency is given by the absence of the inspection equipment superfluous in flight (ie: no chairs, contained dimensions appropriate for the equipment, not for staff).

b) The ability to transport spare parts, additional equipment, land, ... is given by the Ground segment, where overcapacity is less expensive than in the Air segment.

c) The long range required by Flight inspection is one of the main characteristics of the UAS, specially the ones big enough for carrying the inspection equipment.

d) The aerodynamic stability is one of the main requirements of all the UAS devoted to observation (which is the case of almost all the civil UAS projects) which requirements are clearly different from the combat aircraft where could be found some aerodynamics instability.

e) Both low noise and vibration levels (adequates for a human environment) becomes less restrictive when talking about electronic equipment.

f)In UAS, most of the systems that generates electrical noise are not present: Data visualization displays, lighting in cabin, galleys, Wc ... etc. So, electrical noise is lower by construction.

g) As in UAS, the payload is just electronic equipment, the electric system capacity is an inherent capacity of the aircraft design.

h) Flight stability at low speed is part of the basic performances of the majority of UAS as its intended missions are the observation of an area during long periods of time.

i) Adaptability of mission payload is part of the research line of ICARUS [ICARUS_4], the research group that generated the present proposal.

j) Environmental control is reduced to the strictly required for the avionics and payload because the flight inspector and the pilot are comfortably sited on the ground system. 
k)The use of autopilot is intrinsic to UAS technology. Additionally, one of the ICARUS group research lines [ICARUS_2] comprises the formal specification of mission and their exploitation. The use of this formalism reduces the workload of the crew comparing with a standard autopilot.

Analyzing briefly the ICAO recommendations [ICAO_1] for flight inspection aircrafts, it seems clear the improvement that results from the substitution of conventional aircraft by UAS. Additional analysis of national regulations as [FAA_1] or European equivalent documents could add more detail into the text, but tha main requirements for the flight inspection aircrafts are quite similar. The applicability of UAS technology to Flight Inspection is determined by the technological/regulatory availability of systems that meet the standards required by the Air Transport System.

\section{Prototype development and test}

The development of UAS for NavAids inspection needs a previous work on a demonstrator system or prototype. Between the issues to prove in this demonstrator we may find three technological aspects: the Sense \& Avoid technology, the interoperability between UAS and ATM, and the mission development. The last one is the target of this first prototype.

In figure 2 could be seen an equivalence for UAV Certification Specification that was presented in [EASA_2] together with a "safety target" approach which has been discarded in the final policy [EASA_3].

\section{CIVIL AIRCRAFT REGULATIONS}

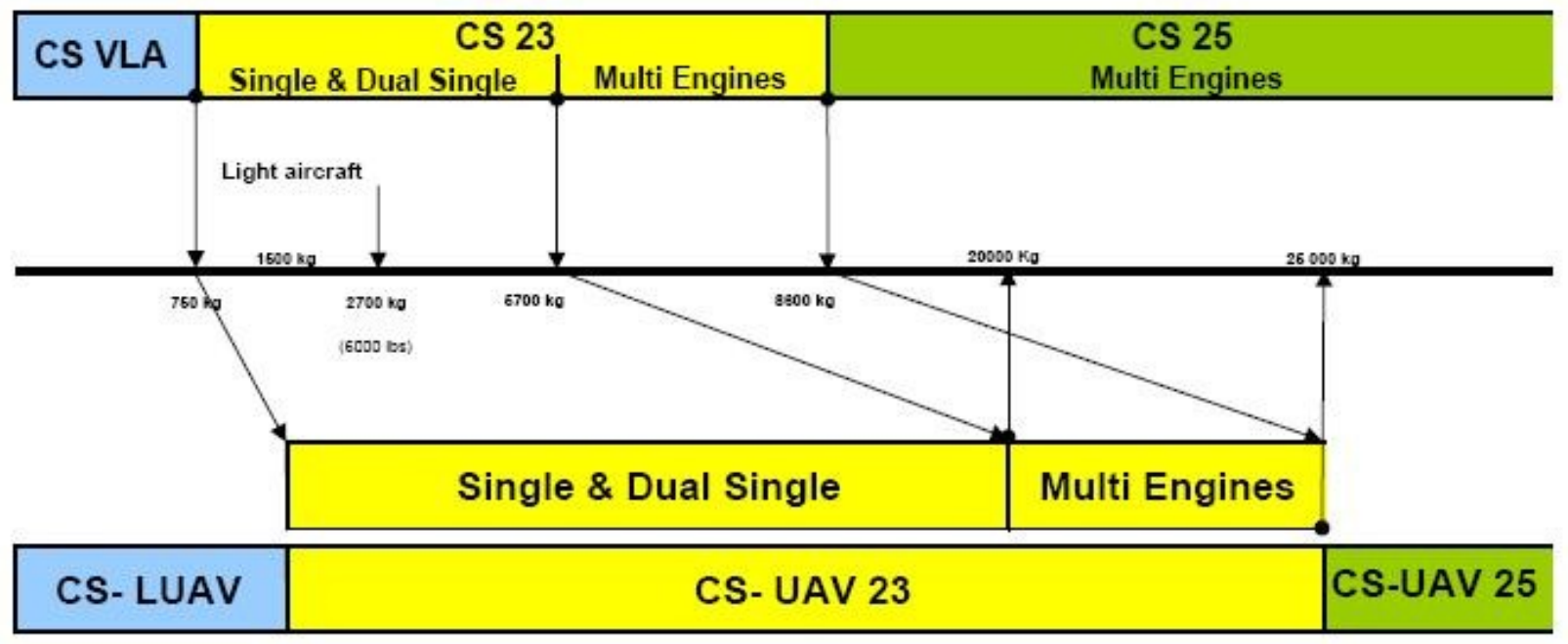

\section{UAV REGULATIONS}

Figure 2: EASA regulation proposed equivalence

Currently EASA is assuming new competencies [EASA_3] that affects the UAS operations:

- air operations; flight crew licensing and third country aircraft. The implementing Rules are expected in 2009.

- airport operations, air traffic management and air navigation services. The implementing Rules are expected in 2010 (approximately)

Further development of the policy relies on several tasks proposed to Eurocae WG 73 (which is coordinated with RTCA) .

As stated by FAA in [EASA_3], an Aviation Rulemaking committee (ARC) has been created and final rule is expected in 2010/2011.

RTCA is working on the definition of Minimum Aviation System Performance Standards (MASPS) for:

- UAS

- Sense \& Avoid (Includes separation assurance)

- Command \& Control

American Institute of Aeronautics and Astronautics 
Terms of reference has been re-baselined to $>2015$.

Due to the lack of effective regulations, and to the need of evolve technically in the same direction than regulations, different initiatives have been started. The Air4all consortium [Air4A_1] proposes an stepped approach starting with synthetic environment simulations, restricted airspace operations and with the objective of final transnational operations in non-restricted airspace.

Adopting a similar stepped approach, first validations of the flight Inspection system is envisaged through the simulation workbench for rapid prototyping of ICARUS [ICARUS_1]. Once this initial validation have been satisfactorily conducted, some flight test are envisaged in restricted airspace. The conditionings of the environment for the prototype test operations should include the use of remote airways, with no other air traffic around. Moreover, a set of different remote environments would be needed for testing the system with different orographic grounds. Preliminary dialog with the regional airport authority of Catalonia has already started and some airports have been preselected for these flight test. These preselected airports are included in the regional government airports strategic plan [GENCAT_1] for its improvement (with additional Navaids), construction or re-opening (an old private airfield that becomes public).

\section{Proposed architecture}

Figure 1 present the architecture of the flight inspection system using a UAS. Given a ground NavAid to inspect we propose the division of the flight inspection functions into two segments: the Air Segment and the Ground Segment. Both inspector and pilot will stand on the ground.
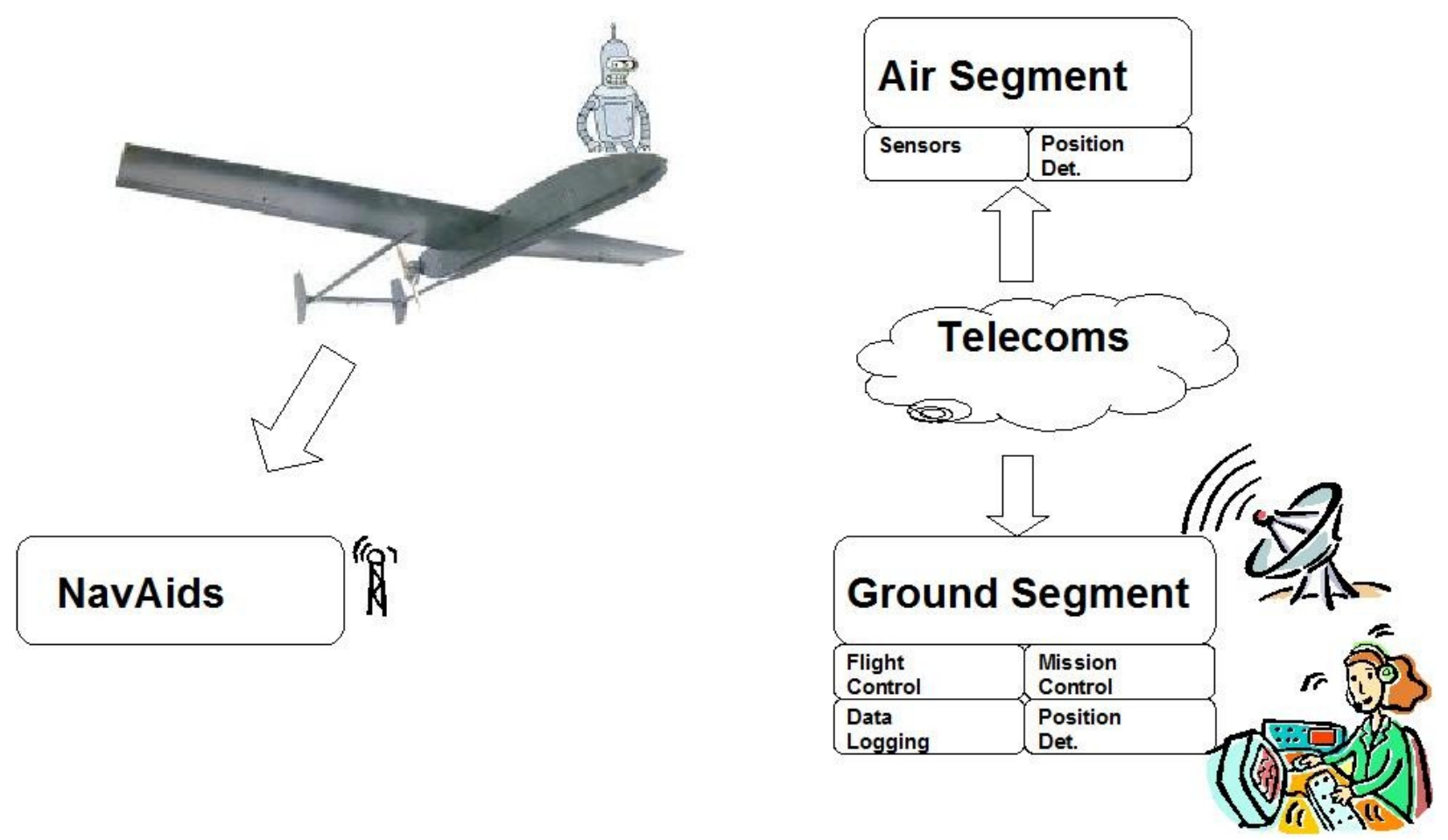

Figure 3: Proposed Flight Inspection System architecture

As seen in figure 3, the flight inspection system has been splitted in two separated segments linked through telecoms: Air Segment and Ground Segment.

- Air Segment. It is kept the essential for flight inspection on the Air Segment: the sensors and the position determination system.

Sensors used are basically the antennae and the receivers of the different Navaids that are inspected (e.g: VOR, DME, ILS). Its function is to measure the values of the Navaid in the air.

$\bigcirc$ Position Determination System. To verify the adequacy of the measured values to the standard, each value needs to be related to the position in which was measured. Usually it is composed by a Satellite 
based navigation system but can also include other means as teodolites.

- Ground Segment. The systems that are not strictly necessary on the Air segment are placed on the Ground Segment.

Flight Control. By Flight Control it is intended the operations that a pilot has to do in a manned aircraft to operate in the airspace.

Mission Control. The control of the Flight Inspection to ensure that the required data are collected and that the flight inspection has been conducted properly.

$\bigcirc$ Data Logging. To comply with the ICAO recommendations it is mandatory the storage of the telemetry and instruments during the flight.

O Position Determination. Some of the position determination systems can be placed on ground (notably the vision based).

- Telecoms. With this architecture, there is a need for rely both segments.

$\bigcirc$ Telemetry. Data from navigation, aircraft status and mission payload has to be downloaded to the ground in order to monitorize the flight inspection.

O Control. Once the telemetry is received, it is necessary to act both over the aircraft and the flight inspection payload.

\section{A) Payload for navaids inspection}

ICAO details in adjoint 1 of chapter 1 of [ICAO_1] a set of recommendations for the Flight Inspection Aircrafts. In paragraph 2 "Aircraft instruments" is described a bloc diagram similar to the presented in figure 3:

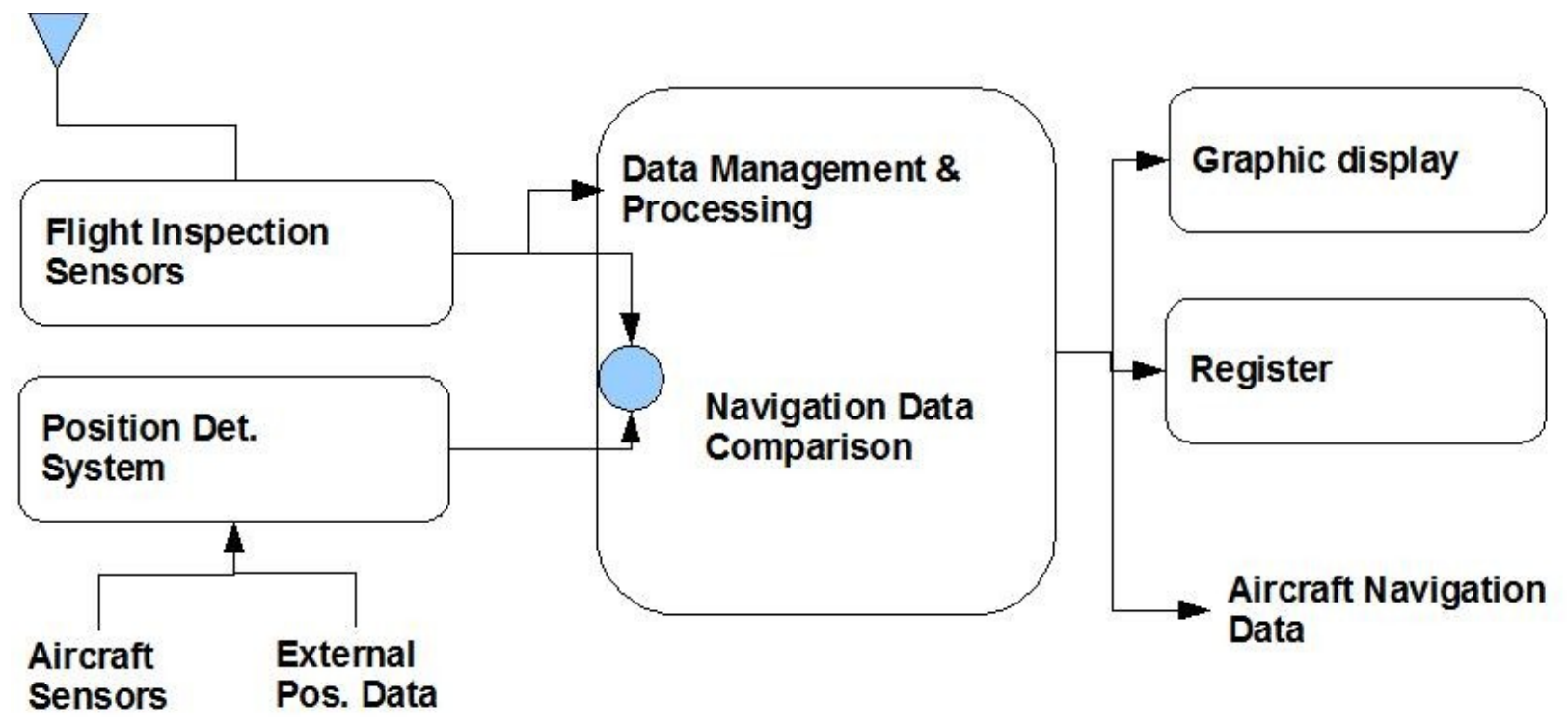

Figure 4: Flight Inspection equipment bloc diagram

Flight Inspection Sensors must provide both navigation data for flight inspection and as for normal use. These sensors could be the same kind of the used for navigation or high quality receptors but it seems more adequate for an UAS architecture to rely in a data acquisition card of a embedded computer that could capture and pre-process the data in order to adapt to the transmission to ground.

Position determination system. The position must be accurately obtained in order to determine the exactitude of the navigation. The data obtained by this system must be compared with the data of the flight inspection sensors.

Processing and display. The cornerstone of this function is to compare the observed behavior with the expected one. That the reason why Position determination is so important in flight Inspection. In current architecture (see fig. 1) both processing and display functions are performed on board, even when the ICAO explicitly says in its doc [ICAO_1], adjoint 1 to chapter 1, paragraph 2.7 that the processing could be done as well in line as in post process. In our architecture (see fig. 3 ) both functions are performed in line but on ground instead that on board.

Data register. Raw data and the results of the inspection must be stored. As the processing and display of the data is retained on ground, the storage of the results is intended to be performed on ground as well as the storage of the 
raw data processed in the flight inspection.

Note that the bloc diagram of figure 4 and the architecture proposed in figure 3 are compatibles thanks to the use of telecommunications. These telecommunications must ensure the integrity of the data (monitor and control data) as well as the resilience of itself against unexpected situations.

\section{B) Service Oriented Architecture}

Service Oriented Architectures (SOA) are getting common in several domains, for example Web Services in the Internet world and UPnP in the home automation area. SOA is an architectural style whose goal is to achieve loose coupling among interacting components or services. A service is a unit of work done by a service provider to achieve desired end results for a service consumer. Both provider and consumer are roles played by software agents on behalf of their owners. The results of a service are usually the change of state for the consumer but can also be a change of state for the provider or for both.

The idea of these architectures is to increment the interoperability, flexibility and extensibility of the designed system and their individual components. In our approach, the UAS, both ground and air segments, are composed by a number of low-cost distributed computing devices connected by a network. The functionality of the system is divided into a set of reusable software peaces, the services, that are distributed over the different computing nodes of the network. A middleware manages their lifecycle and their communication, operating the UAS as a Distributed Embedded System. All services operate at the same level, on top of the middleware, which mainly offers publishsubscribe primitives (see Figure 4). Additional efforts are needed because of the specificities of the UAS domain, in particular, when managing communications between air and ground segments. The service oriented middleware has the capacity of interoperating with unreliable and high-latency point-to-point networks.

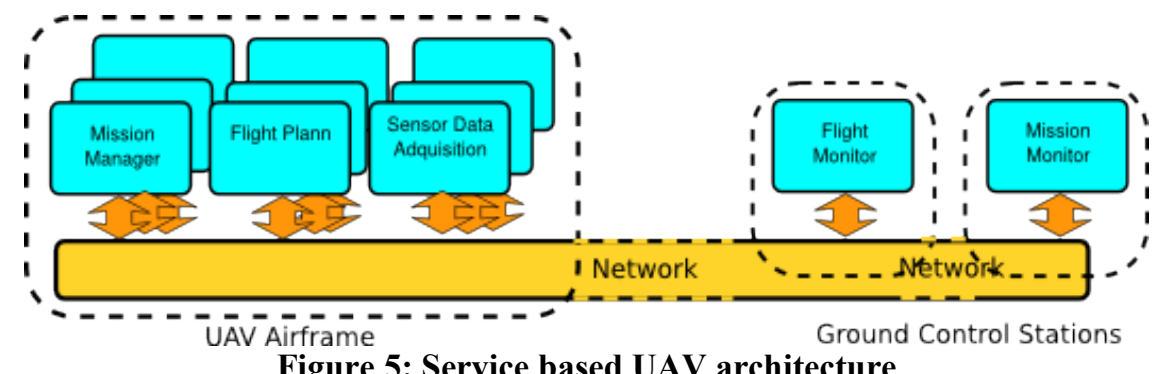

Figure 5: Service based UAV architecture

Services describe themselves on request, sending descriptive messages explaining its operation and its capabilities. These messages define the structure and semantics of the services provided, known as interface. These features are inspired on the object oriented paradigm, which strongly suggests that you should bind data and its processing together. When some service needs an external functionality, it broadcast a request for services descriptions. If another service of the system has the needed functionality, the former subscribes to this provider service using its common interface.

We have designed a software layer that allows the development of complex and collaborative services. The main objective is to obtain ease to reuse services for several UAV applications. The available modules in our UAV provide an extensive set of services, covering an important part of the generic functionalities present in many missions. Therefore, to adapt our aircraft for a new mission a simple reconfiguration is needed, without adding any new software or hardware.

\section{C) Mission Services for Inspections}

We have developed the middleware layer that supports this Service Oriented Architecture and now we are developing a first collection of services. The minimum common set of services needed for most UAV missions are related with flight features. They are not the target of this paper and we will simplified them with the Flight Plan Manager service. This is a service that summarizes the fight capabilities of the UAV. It interacts with the Flight Control System (FCS) providing real time telemetry at a given rate, dispatching alarms in case of failure and entering the WayPoints of the mission flight. 


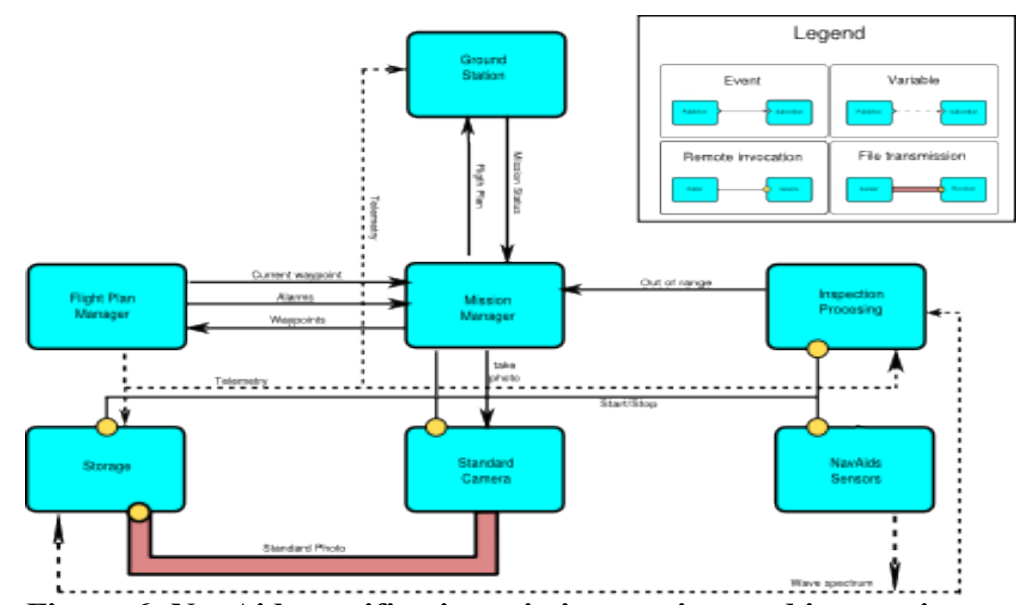

Figure 6: NavAids certification mission services and interactions

Figure 6 shows an example of the main services for a NavAids inspection mission. To interact with the Flight Plan Manager we define a Mission Manager service. This is the director service. It may redesign the flight pattern in case inspection requests it. The Mission Manager is also in charge of starting/stopping payload.

We define two payload devices: a camera and and oscillator that measures the NavAids radio frequencies. The camera is only for situation information, but the oscillator is crucial for the mission. Both devices have a service that publish their capture data.

The Inspection service subscribes to the flight Telemetry, available from the Flight Plan Manager, and it subscribes also to the oscillator Frequency. With both data the Inspection service validates the errors limits and it decides if there is a certification problem. In case of an out of range difference, the Inspection service notifies the Mission service, which may decide that the flight must be repeated. Finally, a Storage service will keep all flight relevant information for post analysis.

\section{D) Air Ground communications}

In a UAV environment it is very common to use different links to support communication at different ranges and to provide redundancy in the case a link is down. These point-to-point links usually do not support multicast and may have associated costs (economical or power-consumption) that restrict their usage to specific situations or very important transmissions. The UAV should be intelligent enough to send the data through the most appropriate channel. This decision should be based on the type and length of the data to send, the current quality of the different links and the mission status. At the same time, the communication between the UAV and the ground station should be as much uniform and transparent to the services as possible in order to allow the deployment of the same services in different missions over different network relays.

In Figure 7, a possible configuration of services in the distributed embedded system is shown. All the services are layered on top the virtual or overlay network. However, this overlay network is divided into three physical networks. Two of them are Ethernet networks: one in the UAV airframe interconnecting all the devices onboard, another one in the ground control station, connecting all the computers that control and supervise the UAV operation. The third one connects the former via a point-to-point link that sends commands from the ground to the aircraft, and telemetry data from the aircraft sensors and cameras to the ground control station. The idea is that any service deployed over this overlay network could access and be accessed transparently for any other service deployed on the same overlay network. The Communication Gateway is the service that implements the continuity of the data communications through the overlay network. 


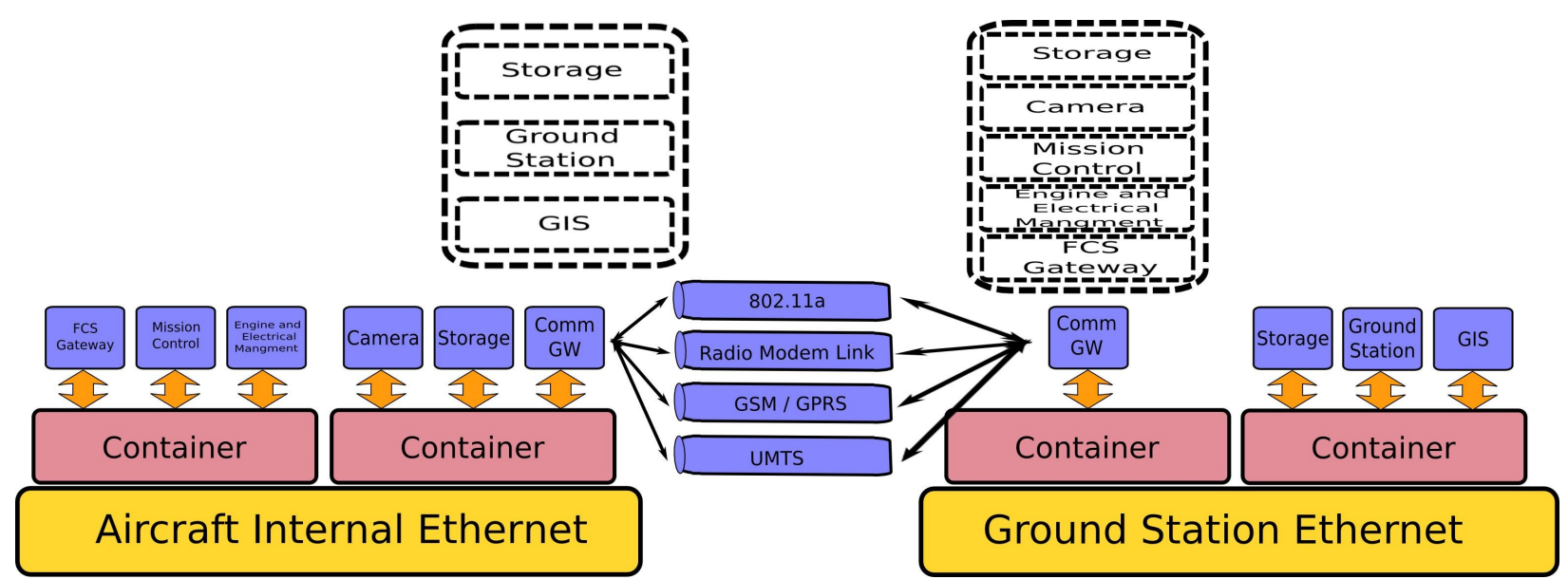

Figure 7: Distributed Embedded system through Gateways

The intelligence needed by the communications system shall be supported not only by the medium access which shall interpret in some manner the content of the different messages but also by the message catalog himself. As shown in [TADIL_1] there is already a catalog with categorized messages: the link 16. This catalog is used by US DoD TActical Information Digital Information Link J (TADIL-J) and by NATO in its Multifunctional Information Distribution System (MIDS). This catalog allows an automatic management of different kinds of information from different sources in a netcentric operation that increase the situational awareness, the resilience of the network and reduces the workload of the flight crew as it could be provided with an integrated picture of the operations scenario. In addition to the automatic management of the information, link 16 also provides a mean to exchange mission data as mission assignments, flight paths or vectors.

MIDS usage has already been investigated in the past by Eurocontrol for it eventual usage in civil aviation [EATM_1] but this study was limited to its use in Surveillance as base for ADS-B. The conclusions of this study were that technically and economically was feasible but due to the simultaneus use of the spectrum it was discarded its common use.

It is declared as an Civil-Military interoperability issue by Eurocontrol in [MILT_1] explaining that the civil use of MIDS networks is very difficult due to the simultaneous radiofrequency spectrum usage.

Finally, as the use of the ICARUS architecture allows the abstraction of the physical network, the adoption of the link 16 catalog (as a base for the development of our set of messages) does not compromise the spectrum usage of military.

\section{Conclusions}

This proposal presents a set of technological developments applied to the inspection of Radio Navigation Aids, but whose predictable impact goes beyond, into the field of civil UAS applications.

We intend to provide the inspection platform with a mission system that allows a dynamic and flexible management of the payload shipped in the Air segment. This flexibility and dynamism is obtained thanks to protocols of platform abstraction [ICARUS_3] and mission definition [ICARUS_2].

The mission definition protocol intends to improve the navigation capabilities of the UAV platform based on leading it beyond the current point to point and segments navigation that allows the aRea NAVigation (RNAV) standard [RTCA_3].

The UAV Abstraction Layer [ICARUS_3] intends to provide better communication management allowing management of communications at high level with an abstraction of the hardware. This UAL allows also gradual improvements on the communication capabilities (increasing bandwith, implementing encryption, modifying physical means...) independently of the management of the payload.

Another technological developments needed in this proposal are the automatic precise positioning systems, whether based on GPS or optical technologies.

Taking advantage of the already innovative avionics developments mentioned above, we intend to develop a new system of testing that would allow us to improve the efficiency of NavAids Flight inspections, creating a new paradigm of Flight inspection.

This paradigm materialize in a new NavAids inspection system through UAS that contribute to optimizing the 
system of air traffic management by providing a more efficient method of conducting NavAids inspections imposed by regulations.

From an operational standpoint, we intend to involve the Navigation Service Provider in the development and integration of UAS through a research from which could get a direct benefit.

Finally, this proposal intends to integrate the mentioned developments in a environmentally more efficient manner than the present, reducing noise pollution and gas from an activity inherent in the air transport system.

\section{Acknowledgments}

This work has been partially supported by :

The Spanish Ministry of Education with grant TIN2007-63927.

The Industrial and Technological development Center with grant SAE-20081098

\section{References}

[ICAO_1] "Manual on Testing of Radio Navigation Aids", ICAO Doc. 8071, $4^{\text {th }}$ edition 2000.

[EATM_1] "Feasibility study for civil aviation data link for ADS-B based on MIDS/Link 16", Eurocontrol TRS/157/02, $5^{\text {th }}$ July 2003.

[TADIL_1] "TADIL J. Introduction to Tactical digital information link J and quick reference guide", Air Land Sea Application center, June 2000.

[MILT_1] “Civil-Military CNS/ATM Interoperability Roadmap”, Eurocontrol, 3th January, 2006.

[Qvist_1] "Remote Flight Inspection of Enroute Facilities", Ian Qvist, Flight Inspector, South African Civil Aviation Authority, 14th IFIS

[Wede_1] "The Future of the Flight Inspection World, A cristall Ball Look into changes ahead, based on current trends and development", Captain Thomas Wede, AFI Flight Inspection GmbH, 14th IFIS.

[SAE_1] "Certification considerations for highly-integrated or complex aircraft systems", Society of Automotive Engineers, Inc. Aerospace Recommended Practice 4754, November 1996

[SAE_2] "Guidelines and methods for conducting the safety assessment process on civil airborne systems and equipment", Society of Automotive Engineers, Inc. Aerospace Recommended Practice 4754, December 1996 1992

[RTCA_1] "Software Considerations in airborne systems and equipment certification", RTCA Inc, DO178B, $1^{\text {st }}$ December

[RTCA_2] "Guidelines for communication, navigation, surveillance, and air traffic management (CNS/ATM) systems software integrity assurance", RTCA Inc, DO 278, March 5, 2002

[RTCA_3] "Minimum Aviation System Performance Standards: Required Navigation Performance for Area Navigation" RTCA Inc, DO-236B $28^{\text {th }}$ October 2003.

[EASA_1] "Extension of the EASA system to the regulation of Air Traffic Management and Air Navigation Services (ATM/ ANS)", Notice of Proposed Amendment (NPA) No 2007-16, EASA, $21^{\text {st }}$ January 2008.

[EASA_2] "Policy for unmanned Aerial Vehicle (UAV) certification", Notice of Proposed Amendment (NPA) No 16/2005, $7^{\text {th }}$ February 2005.

[EASA_3] "Unmanned Aircraft Systems: Considerations for Certification and Interoperability", U.S./ Europe International Aviation Safety Conference, Doug Davis, FAA, and Yves Morier, EASA, $5^{\text {th }}$ June 2008

[FAA_1] "United States Standard Flight Inspection Manual", Departments of the Army, the Navy, and the Air Force and the Federal Aviation Administration, Department of the Army Technical Manual TM 95-225, Department of the Navy Manual NAVAIR 16-1-520, Department of the Air Force Manual AFMAN 11-225, Federal Aviation Administration Order 8200.1C, October 2005

[UAVNET_1] "25 Nations for an Aeronautics Breakthrough, European Civil Unmanned Air Vehicle Roadmap", UAVNET, http://www.uavnet.com $22^{\text {th }}$ Mars 2005.

[AEC_1] "STAR21 - Strategic Aerospace Review for the 21st Century" European Advisory Group on Aerospace European Commission, Enterprise Directorate-General, Brussels, Belgium, July 2002

[EuroC_1] "Unmanned Aircraft Systems, UAS ATM Integration Activity Stream 1 Outline", Holger Matthiesen EUROCONTROL UAS ATM Integration Activity Manager, the Eurocontrol UAS ATM integration workshop May 7-8, 2008

[9] http://avnwww.jccbi.gov/icasc/ fi_history_general.html

[IFATS_1] "Innovative Future Air Transport System. The IFATS project description, at a glance", Consortium IFATS November 2004 -Web Site : http://www.ifats-project.org

[SESAR_1] "Work Programme for 2008-2013", SESAR ExCom 22st Decision Note- Ref: MGT-083-002-01-00, Brussels, $15^{\mathrm{th}}$ May 2008.

[Volpe_1] "Vulnerability Assessment of the U.S. Transportation Infrastructure that Relies on GPS."Carroll, James, Van Dyke, Karen, Kraemer, John and Charles Rodgers. 2001. ION National Technical Meeting, Long Beach, CA, January 22-24, 2001.

[Air4A_1] "UAV Insertion into General Air Traffic", AIR4ALL Consortium, Air4All $3^{\text {rd }}$ Stakeholder 
Workshop, Brussels $23^{\text {rd }}$ June 2008.

[ICARUS_1] "Service Oriented Fast Prototyping Environment for UAS Missions", P. Royo, J. López, J. Tristancho, J. Lema, B. López and E. Pastor, Technical University of Catalonia (UPC), Castelldefels, Barcelona, Spain" $8^{\text {th }}$ January 2009

[ICARUS_2] "Mission Aware Flight Planning for Unmanned Aerial Systems.", E.Santamaria, P.Royo, C.Barrado, E.Pastor, J.López, X.Prats. AIAA Guidance, Navigation and Control Conference, Hawaii 2008.

[ICARUS_3] "Service Abstraction Layer for UAV Flexible Application Development", P.Royo, J.Lopez, E.Pastor, C.Barrado.. 46th AIAA Aerospace Sciences Meeting and Exhibit, Reno, Nevada 2008.

[ICARUS_4] "Modular Avionics for Seamless Reconfigurable UAS Missions", J.Lopez, P.Royo, C.Barrado. 27th Digital Avionics Systems Conference, Minesota 2008.

[GENCAT_1] "Pla d'aeroports, aeródroms I Heliports de Catalunya 2007-2012” Secretaria de la Mobilitat, Direccio General de Ports Aeroports I Cosets, Generalitat de Catalunya. www.gencat.net, $5^{\text {th }}$ December 2007 